from PHE to key stakeholders. This project will inform the development of a knowledge mobilisation strategy at PHE.

\section{P70 ARE SEPSIS AWARENESS AND ANTIMICROBIAL STEWARDSHIP COMPETING GOALS? A CONTENT ANALYSIS OF THE FRAMING OF SEPSIS AND ANTIMICROBIAL RESISTANCE IN THE POPULAR NEWS MEDIA}

U Rush*, S Hilton, LM McDaid, C Patterson. CSO/MRC Social and Public Health Sciences Unit, University of Glasgow, Glasgow, UK

\subsection{6/jech-2017-SSMAbstracts. 171}

Background Antimicrobial resistance has emerged as one of the greatest threats to population health of modern times. A review on antimicrobial resistance in 2016 by the UK government predicted that infections caused by resistant organisms could be responsible for ten million deaths annually by 2040 . A key driver of resistance is inappropriate use of antibiotics within human healthcare in managing minor illnesses that would resolve spontaneously without drug treatment. This has been referred to as 'just in case' prescribing.

The Chief Medical Officer, Dame Sally Davies, has called for a reduction in unnecessary prescribing as part of improved antimicrobial stewardship however; there is evidence that antimicrobial resistance remains overlooked by the public and by health professionals as an important health risk.

Antimicrobial stewardship is complicated by growing recognition that serious bacterial infections can initially present in the same way as mild viral illnesses. Recently, there has been a drive to improve recognition and management of sepsis, a serious consequence of untreated bacterial infection that is associated with high mortality. Simultaneously, there has been a large volume of high profile stories about sepsis in the media. This study aims to explore similarities and differences in how antimicrobial resistance and sepsis are framed in the popular print news.

Methods Quantitative analysis of the manifest content of 297 articles about sepsis and 163 articles about antimicrobial resistance published in 11 UK national newspapers on all available dates until 31 st December 2016, identified via a systematic search on the Nexis database. A coding frame was developed through a priori knowledge and close reading of 200 articles using the constant comparative method. A random sample of $10 \%$ of these articles were coded by two reviewers to ensure consistency in the application of codes. The remaining articles were coded by a single reviewer and analysed using SPSS.

Results Articles about sepsis were more likely to identify individuals who had suffered serious adverse health effects, often infants or young children, and to criticise the actions of individual health professionals. These factors have been identified as 'media triggers' that can increase public interest in a news story.

Conclusion Exposure to news stories about sepsis has the potential to alter public awareness and perception of risks associated with minor illness. This may impact on expectations of receiving antimicrobial treatment, with implications for antimicrobial stewardship.

\section{P71 IMPLEMENTATION OF STEP-DOWN INTERMEDIATE CARE IN GLASGOW CITY: A QUALITATIVE STUDY EXPLORING BARRIERS AND ENABLERS}

KA Levin*, EM Crighton. Public Health Directorate, Glasgow, UK

\subsection{6/jech-2017-SSMAbstracts. 172}

Background The aim of Step-down Intermediate Care (IC) is to provide a short-term care environment for older people ready for discharge from acute hospital but requiring a period of assessment and/or rehabilitation. There are a number of models of IC in the UK. Glasgow City's model of IC has gone through several iterations and stages of development. This study aims to examine the implementation of IC in Glasgow City, to identify enabling factors and barriers.

Methods The study used multiple qualitative methodologies: document review, semi structured interviews, focus groups and attendance at IC development and care home review meetings. The documentary analysis included reports and meeting minutes. Nine key members of staff were interviewed and three focus groups were run; the first included IC social work staff from Glasgow City's three sectors, the second included rehab staff from two sectors, and the third included care home staff from three IC units. Framework analysis was used to identify common themes.

Results Perceived enablers common to all staffing groups included: buy-in from a range of stakeholders, strong leadership and a risk management system in place, good relationship, trust and communication between agencies, the role of targets and in particular the 72 hour discharge target, training of staff, changing perception of risk and risk aversion among practitioners, the right infrastructure and staffing in place, an accommodation based strategy for patients discharged from IC, the right context of political priorities, funding, and a wider model of care for older people, and ongoing adaptation of the model in discussion with frontline staff. Perceived barriers included: differing perception of IC aims, the use of separate technologies by agencies for recording information, transition of patients from hospital to IC, inappropriate referrals to IC, and variation in health and social care systems between sectors and hospitals. Additionally, perceptions differed by staffing group. While social workers noted a need for continuous education of acute staff, and placement issues on discharge from IC, rehab staff found that care homes often lacked appropriate facilities. Both social work and rehab staff noted the benefits of being attached to one unit, while care home staff described the importance of continuity of acute staff.

Conclusion The proposed benefits of IC were understood and supported anecdotally by staff. The development of IC in Glasgow gives an insight into enablers of and barriers to implementation of the service, highlighting further opportunities for improvements to the model. 